\title{
Poliorcética, morfología edilicia y técnicas constructivas en el Tossal de la Vila, un recinto fortificado de época emiral en el extremo septentrional del Šarq al-Andalus
}

Poliorcetics, architectural morphology and construction techniques at Tossal de la Vila, a fortified enclosure from the Emirate period in the northernmost end of Šarq al-Andalus

\author{
Joan Negre a ${ }^{\text {, Ferran Falomir }}{ }^{\text {b }}$, Marta Pérez-Polo ${ }^{\text {, }}$, Gustau Aguilella ${ }^{\text {d }}$ \\ ${ }^{a}$ Universitat Autònoma de Barcelona, Bellaterra / Museu Arqueològic de Gandia, Gandia, Spain, joan.negre@gandia.org \\ ${ }^{\text {b }}$ Servei d'Investigacions Arqueològiques i Prehistòriques, Castelló de la Plana, Spain, ffalomir@ dipcas.es \\ ${ }^{\mathrm{c}}$ Universidad de Navarra, Pamplona, Spain, marta.perez.polo@ gmail.com \\ d Servei d'Investigacions Arqueològiques i Prehistòriques, Castelló de la Plana, Spain, gustauaguilella@ dipcas.es
}

\begin{abstract}
This work focuses on the first results from the systematic excavation of the Tossal de la Vila (Serra d'en Galceran, Castelló) archaeological site. This is, a hillfort build during the Emirate of al-Andalus in the intersection between the territories of Tortosa, Valencia and the Iberian System mountain ranges. Our case study is framed within the historiographic discussion on the subject of rocky and castellated settlements in this area set forth thirty years ago by André Bazzana. A debate that was largely enriched by several works pending the last years on the subject of hilly occupations between Late Antiquity and Early Middle Ages. In that direction, we analyse here the architectural questions that have been raised by the recent archaeological works at the site. Specifically, we will try to systematise the different defensive solutions adopted on the design of the fort, as well as the diverse construction techniques used along the fortbuilding process.
\end{abstract}

Keywords: Al-Andalus, fortification, dry stone masonry, incastellamento.

\section{Introducción}

El yacimiento del Tossal de la Vila se sitúa en una de las cimas más preeminentes al sur de la sierra d'En Galceran, a $954 \mathrm{~m}$ de altura (Fig. 1), abarcando una amplia plataforma rocosa cercana a los $5000 \mathrm{~m}^{2}$ de extensión, con verticales inaccesibles por su lado este pero con accesos más suaves desde el resto de direcciones. La cadena montañosa donde se sitúa el sitio arqueológico presenta una orientación catalánide, delimitada al sureste por el corredor de Les Coves -a través del cual discurría la antigua Vía Augusta- y al noroeste por la rambla Carbonera -que circunscribe el paso andalusí desde la costa hacia Alca- ñiz-. Se trata, por tanto, de un enclave estratégico en la bifurcación de las vías que, desde la ciudad de Valencia, se dirigían hacia Tortosa y Zaragoza (Negre, 2013).

El objetivo inicial del trabajo reside, pues, en delimitar el origen de la fortificación, su morfología y desarrollo constructivo, y sus posibles funciones, con el fin de integrar este ejemplo dentro de su problemática historiográfica específica. En este caso, la de la ocupación y fortificación de ciertos enclaves situados en altura entre la Antigüedad Tardía y la Alta Edad Media, un 
proceso que presenta paralelos claros dentro del Occidente mediterráneo (Schneider, 2001; Catalán, et al., 2004; Gibert, 2018). Se trata, en cualquier caso, de una dinámica histórica compleja que responde a realidades sociales y políticas diversas, con múltiples causas en su génesis, así como en la morfología y funciones de los distintos elementos que componen su plasmación material en el territorio. Es por ello que se han propuesto lecturas complementarias para explicar el surgimiento de estos enclaves y su papel en la historia: como centros de poder local (Schneider, 2001, pp. 436-439; Castellanos, Martín Viso, 2005), como puntos de control viario (Schneider, 2001, pp. 440-442; Bazzana, 1992, p. 277) o incluso como asentamientos vinculados a comunidades campesinas en un intento de escapar del control de los principales poderes territoriales o de las consecuencias de la conquista de al-Andalus (Acién, 2001, p. 60; Schneider, 2001, pp. 439-440), entre otras.
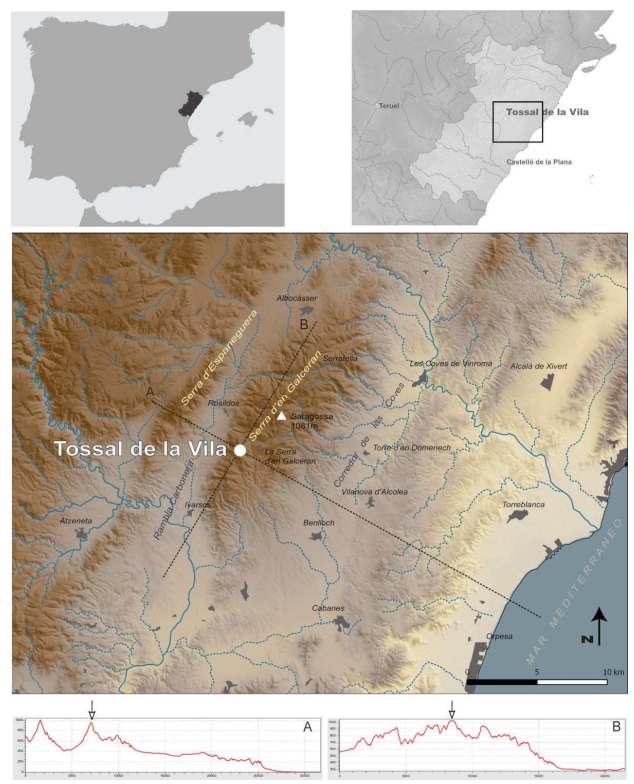

Fig. 1. Contextualización geográfica.

En el caso específico del extremo septentrional del Šarq al-Andalus, es a André Bazzana a quien debemos la síntesis de esta problemática (Bazzana, 1992, pp. 273-275), aunque su desarrollo ha quedado abandonado durante las últimas décadas. Fue el arqueólogo francés quien propu- so una primera identificación de los principales asentamientos en altura en esta región, claramente diferenciados de otro conjunto de recintos, descritos como enigmáticos en sus trabajos (Bazzana, 1992, pp. 348-353).

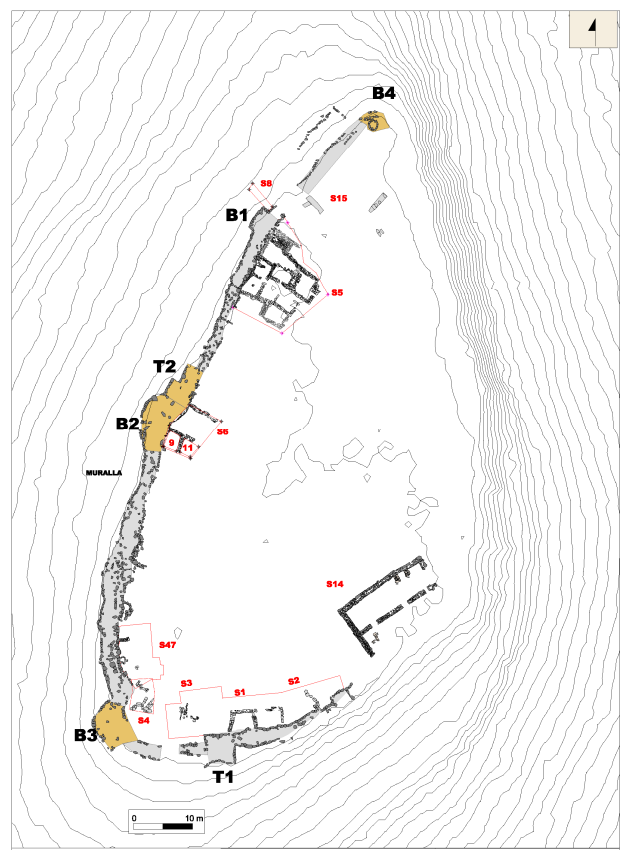

Fig. 2. Planta general del yacimiento (2019).

En el primer grupo destacaba los casos de Mollet y Marinet, para los cuales aventuraba funciones diferenciadas: las propias de un recinto militar vinculado al control viario y territorial (Bazzana, 1992, pp. 277-278), y las de un asentamientorefugio de poblaciones campesinas (Bazzana, 1992, p. 280), respectivamente. Del segundo grupo tan solo podía definir una serie de características comunes (sitios en altura con difícil acceso, construcción con mampostería en seco, muro perimetral adaptado a las curvas de nivel, inexistencia de estructuras habitacionales o un registro cerámico muy escaso), aunque proponía ya una vinculación con actividades de tipo pastoril, como las atribuidas a los sitios castellonenses de la Garrotxa (Cervera del Maestrat), Subarra (Benlloc) o el Punt del Cid (Almenara), a los que se agregaron nuevos casos en años sucesivos (Bazzana, 1992, pp. 351-353). 
De esta manera, el proyecto en que se enmarca este trabajo busca, como resultado final, analizar y describir con claridad este proceso histórico, a través de la excavación en extensión de algunos de los ejemplos identificados, el sondeo de otros, y el estudio pormenorizado de su registro arqueológico. En combinación con estas tareas, se están obteniendo series completas de dataciones absolutas con las que fijar cronológicamente sus secuencias estratigráficas, fases constructivas y contextos productivos. Todo ello con el objetivo de definir, en última instancia, el origen, morfología y funciones de estos recintos, información necesaria para encuadrarlos correctamente dentro de las dinámicas de control territorial en este área (Negre, Suñé, 2019).

No entraremos en detalle, por tanto, en el desarrollo estratigráfico de la secuencia identificada en este yacimiento, que ya ha sido tratada de forma más extensa en otra publicación (Negre $e t$ $a l$., en prensa). Baste decir que han sido documentadas hasta seis fases estratigráficas claramente diferenciadas. Las tres primeras están asociadas a un hábitat protohistórico que culmina en un potente asentamiento del Bronce Final/Hierro Antiguo fechado entre los siglos VIII y principios del VII AEC (Aguilella, et al., 2016). Tras una larga desocupación, las siguientes fases estratigráficas se desarrollan en un momento indeterminado entre el siglo VIII hasta inicios del siglo X. ${ }^{1}$

\section{Diseño del recinto y poliorcética}

El planteamiento topográfico del asentamiento se ve absolutamente mediatizado por el trazado de sus elementos defensivos. El lado de poniente, fácilmente accesible, se fortifica mediante torres y bastiones, cuadrados o de tendencia circular, asociados a una potente muralla que alcanza los 3,50 $\mathrm{m}$ de anchura media gracias a su técnica constructiva que desarrolla la construcción de dos potentes paramentos de mampostería en seco con el espacio entre ellos relleno de bloques y gravas. Su trazado se verá condicionado en primer lugar por la existencia de las estructuras defensivas ya amortizadas del periodo protohistórico y posteriormente por el carácter intermitente de su ocupación, con abandonos ordenados y ocupaciones intermitentes que modificaran sus estructuras defensivas repetidamente en un grado que está en vías de análisis en cuanto a intensidad y funcionalidad.

Previamente a los trabajos de limpieza de la parte exterior de la muralla, se efectuó un sondeo adosado a la parte exterior de B1. Este se planteó transversalmente para detectar posibles líneas no visibles de estructuras exteriores al recinto asociadas a la muralla. La excavación determinó como el desnivel topográfico se solucionó mediante un aterrazamiento que combinó, sobre un primer nivel de bloques más grandes, un segundo nivel formado por una mezcla de bloques de diferentes dimensiones y, finalmente, una unidad de gravas con tierra sobre la que se asentó la muralla.

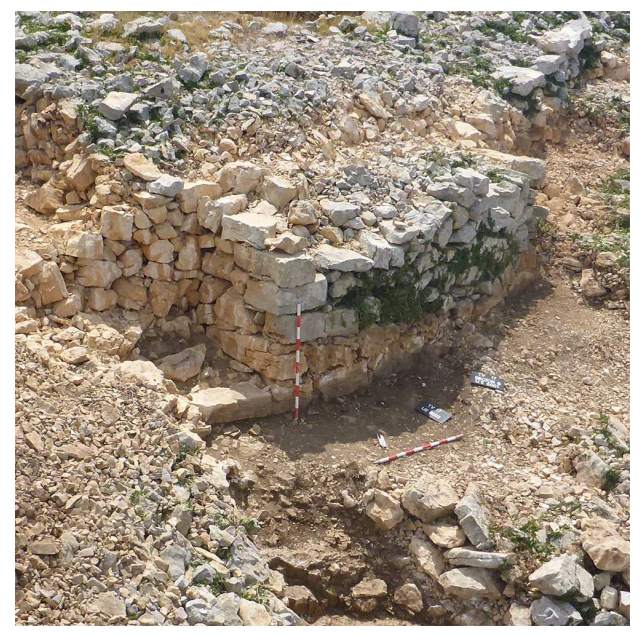

Fig. 3. Excavación del aterrazamiento de cimentación para la construcción de uno de los bastiones de la muralla (B1), articulado con el lienzo.

El muro perimetral del recinto se ve escalonado en sus puntos débiles por dos torres ( $\mathrm{T} 1$ y T2), de planta cuadrangular y adosadas a la muralla, y cuatro bastiones (B1, B2, B3 y B4), de superficie rectangular o de tendencia circular, en los extremos rompientes del muro, menos sobresalientes que la torre. La diferenciación morfológica y de funciones de estos apéndices, así como su evolución diacrónica, que resulta evidente en la transformación de T2 en B2, continúa siendo uno de los elementos clave a resolver durante las 
próximas intervenciones. De la misma manera, sigue sin documentarse la entrada al recinto, manteniéndose la hipótesis abierta en anteriores publicaciones que apunta a un acceso por el extremo sur, entre B3 y T1, o bien recorriendo la muralla hacia el norte para acceder al recinto a través del espacio de flanqueo del bastión septentrional (B4).

\section{Morfología edilicia}

En cuanto a la estructuración del espacio interior del recinto, delimitado entre esta muralla y el acantilado que define su lado oriental, tan solo tenemos constancia de construcciones en los espacios sujetos al resguardo de la muralla, actuando esta como paramento interior. Todo el espacio central del asentamiento aparece libre de construcciones y en el estado actual de la investigación se han reconocido tres espacios con diferentes módulos constructivos (Gutiérrez, 2012). Sólo disponemos de indicios sobre estructuras exentas en el sector 15 , con lo que el acceso a los módulos constructivos se realizaría desde el gran espacio central del asentamiento.

\subsection{Módulos simples anexos a la muralla}

Son las unidades básicas documentadas y corresponden a edificios rectangulares adosados ortogonalmente al recinto defensivo y que pueden tener asociados, conectados mediante puertas, otros módulos simples adosados en sentido longitudinal. En estudios anteriores ya se destacó cómo los sectores 1-3 constituyen el único espacio donde conocemos los volúmenes originales de este tipo de modulación dado que no sufrieron ulteriores modificaciones (Negre et al., en prensa, Fig. 7). Todo el espacio construido en este sector se asocia a la parte interna de la torre de la muralla (T1) y cuenta con varios ámbitos vinculados a un espacio cubierto cuadrangular accesible desde una antesala rectangular en origen enlosada y descubierta (ámbitos $1.1 \mathrm{y}$ 1.2). A este núcleo habitacional se le adosan, de forma longitudinal, unos espacios de planta más o menos rectangular que podrían formar parte de sucesivas ampliaciones, configurados probablemente mediante una técnica mixta de grandes bloques de piedra para las paredes medianeras, combinados con estructuras de madera en su alzado y en el cierre hacia el gran espacio abierto central del recinto.

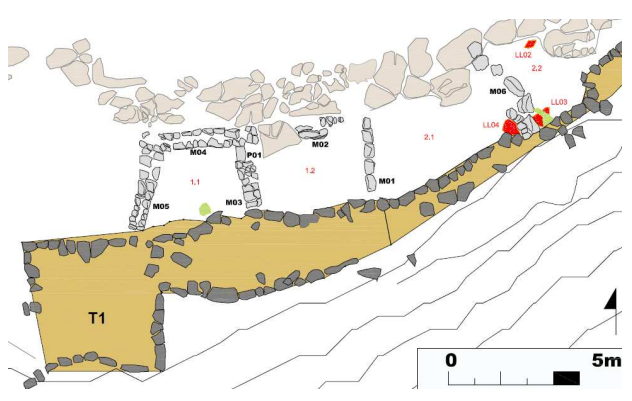

Fig. 4. Sectores 1-3, adosados a la muralla y torre.

La hipótesis que planteamos en su momento pasaba por asociar este sector a un área de almacenamiento y estabulación, donde el espacio principal cubierto tendría la función de despensa o granero debido, entre otros factores, a la falta de estructuras domésticas en su interior y a su sistema de cierre mediante muros de doble paramento. Respecto al resto de ámbitos descubiertos y cercados con estructuras perecederas, proponemos su identificación como áreas para la cabaña ganadera o zonas de ocupación doméstica puntual, dada la existencia, además, de hogares y estructuras de combustión.

Documentamos también restos de este tipo de articulación espacial en pequeños módulos simples en los sectores 6, 9 y 11. En este caso planteamos en su momento la posibilidad de una evolución en estos sectores condicionada por las reformas en el recinto defensivo, dado que existen indicios de la modificación de una torre cuadrada previa (T2) que se verá integrada en un nuevo bastión (B2). Esta remodelación condicionó los edificios previos adosados a ella (Negre, et al, en prensa) (Fig. 8).

En general, la construcción de todos estos módulos se realiza mediante muros de dos o tres hiladas regulares, a modo de zócalos, perpendiculares a la muralla y realizados con doble paramento relleno de grava, de entre 50 y $60 \mathrm{~cm}$ de grosor y unos $50 \mathrm{~cm}$ de altura. El alzado de sus muros acabaría con una tapia de tierra, tal y como se ha podido documentar a través de la 
excavación de los niveles de derrumbe en los distintos ámbitos.

Por lo que respecta a los lienzos internos de los módulos simples en contacto con la muralla, podemos apreciar distintas soluciones: en los sectores 1-3 el acabado se realiza con grandes bloques con hiladas muy irregulares que podrían ser los restos del paramento protohistórico; en cambio, tanto en el sector 9 como en el 5, llama la atención el uso de un paramento a modo de forro, realizado con una mampostería careada con tendencia a la regularidad de hiladas y tamaños de los bloques.

\subsection{Hipótesis de crecimiento modular: unidad compleja estructurada en torno a un patio}

En el sector 5 se identificó ya en 2015 los restos de una trama espacial que denotaba, a pesar de la escasa extensión documentada, la diferencia formal con los módulos simples excavados en los lienzos sur y oeste del recinto. En las sucesivas campañas, pudimos confirmar que en este espacio del asentamiento contábamos con un edificio complejo en el que se reflejaba la evolución morfológica de sus estructuras de hábitat.

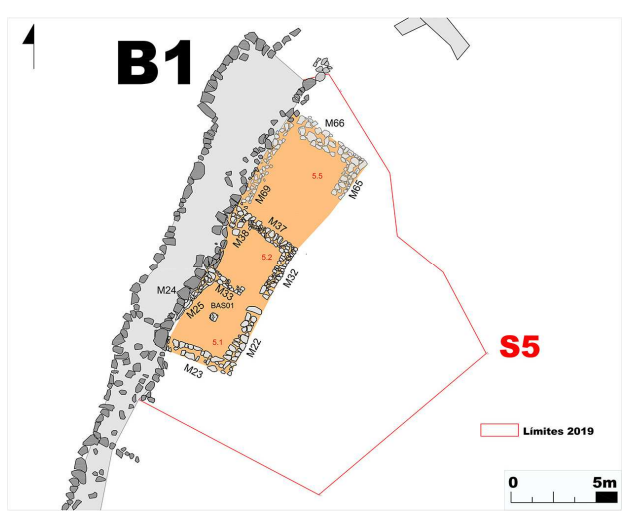

Fig. 5. Primera fase, módulos asociados.

De esta manera, el sector 5 nos permite analizar este proceso desde un estadio donde encontramos módulos simples anexos a la muralla (Fig. 5, ámbitos 5.1, 5.2 y probablemente 5.5), hasta un momento en el que se desarrolla un sistema modular pluricelular mucho más complejo y no documentado en ningún otro sector del recinto hasta el momento.
En la primera de las configuraciones, los ámbitos 5.1 y 5.2 aparecen comunicados, contando con una base de poste en el primero de ellos. Por su parte, el ámbito 5.5 presenta un amplio acceso y su función está aún por determinar. Esta fisonomía aparece levemente modificada en un segundo momento en el que se construye un muro de refuerzo al muro septentrional del ámbito 5.5.

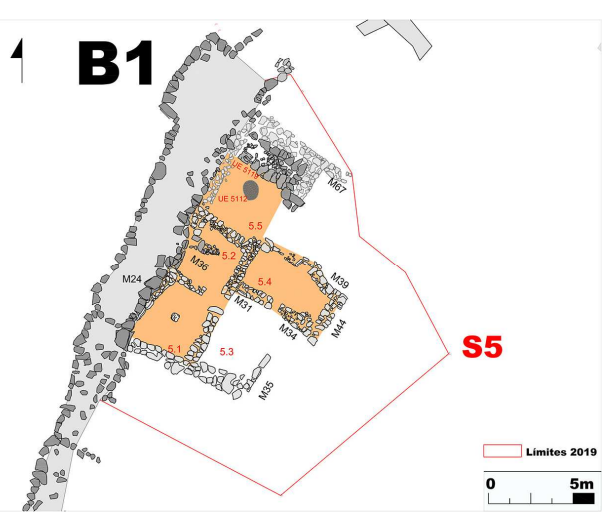

Fig. 6. Segunda fase, módulos agregados.

No muy alejado en el tiempo constatamos la primera transformación de los módulos simples a un espació mas complejo (Fig. 6) con la construcción de dos ámbitos perpendiculares a los primeros y que configuran ya un edificio con un patio de entrada descubierto (ámbito 5.3). Desde allí se accede a los ámbitos 5.1-5.2 y a 5.4, todos ellos dotados con niveles de uso con pavimentos que reaprovechan materiales constructivos del periodo protohistórico, así como con cubiertas vegetales tapadas por losas de piedra, que protegen diversas estructuras asociadas probablemente al almacenaje (M36).

En este momento documentamos cómo el ámbito 5.5 reduce su espacio mediante la construcción de un nuevo refuerzo estructural (UE 5119), y algunos indicios de vida doméstica, hecho que nos lleva a plantear una posible reconfiguración en su uso. También su ubicación, ahora desligada del resto de ámbitos por la construcción de 5.4, invita a sugerir este cambio en cuanto a su articulación espacial y funcional.

Sucesivo a este estadio, y sin solución de continuidad, el edificio se dota de nuevos espacios 
antes de abandonarse, vertebrados por la duplicación del patio (adhesión de 5.8 a 5.3), y ve aparecer a su alrededor, en diferentes alas, sucesivos ámbitos que definen con mayor exactitud su funcionalidad. En el ámbito 5.7, por ejemplo, con entrada independiente desde el área central del yacimiento, aparece un horno elevado (EC02) construido mediante losas y diversas estructuras de combustión a su alrededor, hecho que nos lleva a identificarlo por el momento como una pequeña cocina.

$\mathrm{Al}$ patio central se accede por una única entrada mediante un corto corredor construido con una sola hilada (M48). La compartimentación en dos ámbitos diferenciados corresponde al último momento de uso, y se realiza mediante un muro reaprovechado de la fase anterior, de solo una hilada, que deja al este un nuevo espacio descubierto (5.8) que es ocupado por una gran estructura de combustión (Fig. 7; LL14). Hacia el sur se ha podido documentar otra ala (ámbito 5.9) alrededor del patio central. Esta parte del edificio está en proceso de excavación y queda pendiente su fisonomía y relación con los espacios adyacentes.

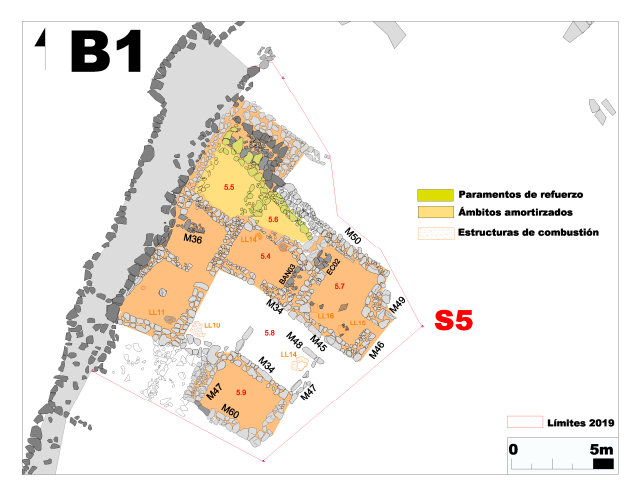

Fig. 7. Fase final, unidad modular compleja estructurada en torno a un patio.

Respecto a la amortización de los ámbitos 5.5 y 5.6, así como al refuerzo estructural detectado en primera instancia en el primero de estos módulos, creemos que hay que relacionarlos con una gran modificación o ampliación del bastión (B1), en aras de reforzar la muralla y facilitar su acceso desde el interior. De esta manera, desde el patio se accedería, al ámbito 5.4, el único que contiene una banqueta asociada (BAN03) y desde el cual se ascendería, con la ayuda de un pequeño umbral o escalón junto a M39, a los módulos amortizados con tierra y bloques $(5.5 \mathrm{y}$ 5.6) y, finalmente, a la muralla.

\subsection{Edificio singular aislado}

En la configuración espacial del recinto destacábamos en anteriores publicaciones la existencia de un edificio singular que, dadas las características que presentaba en cuanto a dimensiones (16 x $9 \mathrm{~m})$, ubicación espacial aislada, técnica constructiva y orientación, asociábamos, a modo de hipótesis, a la existencia de una posible mezquita. En especial destacábamos sus similitudes con otro edificio de estas características ubicado también en un recinto fortificado de gran envergadura, como es el Molón de Camporobles (Lorrio, Sánchez de Prado, 2008, pp. 147-149) En la actualidad está en proceso de excavación, por lo que tan solo hemos podido constatar las similitudes morfológicas con aquella.

Con una planta rectangular (Fig. 8) orientada perfectamente a sudeste cuenta con una nave longitudinal que se abriría a un espacio delantero de iguales dimensiones a modo de patio delantero. El edificio se define como un único módulo construido mediante tres paramentos de una amplitud media de 1,10 m y sin oberturas (M70, M71 y M72) de los cuales el más oriental no se ha conservado en su totalidad.

El muro occidental M70 tiene unas dimensiones de $9 \mathrm{~m}$ de longitud y suponemos que M72 podría haber continuado en paralelo a este hasta cerrar la nave principal, que con una área cercana a los $66 \mathrm{~m}^{2}$. En cuanto al muro suroriental, la qibla de confirmarse nuestra hipótesis, se cierra con otros cuatro paramentos (M73, M75, M76 y M77), que describen tres hiatos en la continuidad del mismo. Los dos situados más al suroeste configuran un espacio cercano a los 1,20 m de amplitud, mientras que el último tan solo de $70 \mathrm{~cm}$.

También podemos constatar cómo durante el proceso de excavación se documentaron varios ortoestatos (Fig. 8, gris claro) situados en las inmediaciones de las dos aperturas más amplias y que de un modo claro habían sido extraídos de su emplazamiento original, creemos que para su 
posterior aprovechamiento en estructuras modernas cercanas al edificio pero que se desecharon por su gran volumen. Todas estas características, sumadas a las múltiples similitudes de este muro con la qibla de la mezquita del Molón, nos llevan a plantear que, en origen, los hiatos murales más amplios debieron ser los mahāàīb (sing. mihrāab) de la mezquita. De planta cuadrada y delimitados por los ortostatos recuperados en sus inmediaciones, no se ha conservado su trazado a causa de su expolio posterior. La entrada a la nave principal, por tanto, se realizaría por el espacio de $70 \mathrm{~cm}$, un acceso de dimensiones más cercanas a las documentadas en el asentamiento.

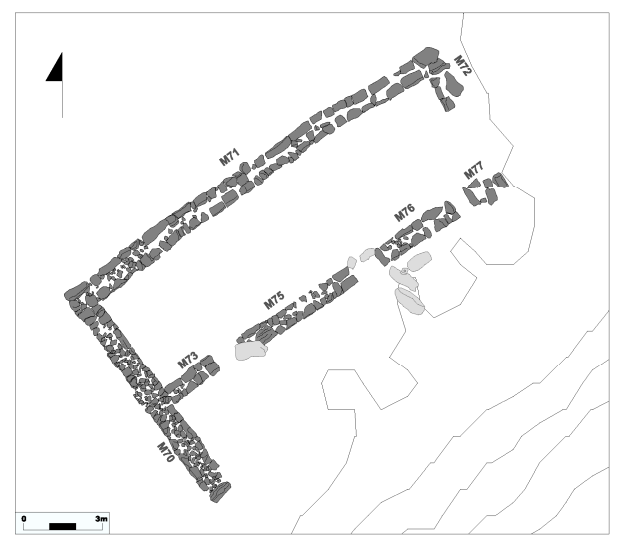

Fig. 8. Edificio singular, posible mezquita.

\section{Conclusiones}

La excavación en extensión del yacimiento arqueológico del Tossal de la Vila supone el primer intento exhaustivo de, al menos, aportar luz a la problemática historiográfica planteada en su día por André Bazzana y nunca resuelta (Bazzana, 1992, pp. 273-287). El proyecto, en el cual se combina la intervención arqueológica sistemática con el estudio crítico de las fuentes escritas árabes y cristianas a nuestra disposición, pretende, a fin de cuentas, complementar la lectura de la organización territorial de los dominios de Tortosa, en los límites de los cuales se situaban estos diversos recintos fortificados en altura (Negre, Suñé, 2019).

Desde la perspectiva arquitectónica, a la cual hemos dedicado este trabajo específicamente, el análisis inicial de los contextos edilicios nos lleva a considerar este recinto como un enclave con clara vocación militar. Una finalidad que giraría en torno al control de unas comunidades rurales en vías de vertebración política y administrativa, pero también con un especial énfasis en la vigilancia de los principales ejes de comunicaciones y encrucijadas, como la que se sitúa a los pies del Tossal de la Vila.

La intermitencia de su ocupación, detectada a través de su secuencia estratigráfica, así como las constantes reformas en sus estructuras de hábitat y almacenamiento, indican una dinámica de uso orientada a establecimientos de duración desconocida pero intermitente por parte de un grupo militarizado, a tenor del registro arqueológico recuperado. Un colectivo, el de sus ocupantes, que aún no hemos identificado, por lo que resulta difícil asociar esta dinámica a una iniciativa estatal, a través de los gobernadores de Tortosa, o a los intentos de algún grupo local por establecer un cierto poder autónomo.

En cualquier caso, resulta evidente que el recinto presenta unas estructuras poliorcéticas potentes, con una muralla de entre 3 y 5 m jalonada con bastiones y torres, cuya planificación y ejecución pensamos que debe atribuirse a un grupo especializado en tales actuaciones. El único sistema edilicio pluricelular muestra también una evolución clara a partir de un módulo simple, un proceso que puede observarse en las distintas adiciones al complejo, no siempre ortogonales al primer espacio y que configuran finalmente un espacio claramente jerarquizado. El hecho de que la cocina sea el único elemento desconectado de este edificio y con entrada directamente desde el exterior también es indicativo de los usos habituales de una guarnición, que en su mayoría estaría instalada mediante estructuras transportables en el espacio abierto central del recinto.

Por último, el único edificio exento identificado presenta unas características de orientación, morfología, factura y monumentalidad que resultan coincidentes con diversas mezquitas rurales excavadas en similares contextos. Sus potentes paramentos, así como el tamaño con el cual contaría su nave principal, a la espera de la 
excavación de su interior durante la próxima campaña de 2020, apuntan, nuevamente, hacia una iniciativa especializada y vinculada, en cualquier caso, a un grupo con un grado de islamización elevado, sobretodo si tenemos en cuenta los momentos iniciales del proceso de asimilación del islam -segunda mitad del siglo VIII y siguiente centuria- en que nos encontramos y el contexto absolutamente periférico del recinto estudiado.

\begin{abstract}
Nota
${ }^{1}$ Las dos primeras dataciones realizadas (Beta419185 y Beta-503127) proceden de restos de madera carbonizada asociadas al momento de colmatación de los hogares LL11 y LL15. En el primer caso marca una horquilla entre finales del siglo IX y toda la siguiente centuria (Cal AD 890-1015), con un pico estadístico vinculado al intervalo marcado por una desviación estándar, que marca una alta probabilidad vinculada al primer cuarto del siglo X. En el segundo, la horquilla oscila entre la segunda mitad del siglo VIII y todo el IX (Cal AD 765-895).
\end{abstract}

\section{Bibliography}

Acién, M. (2001). "De nuevo sobre la fortificación del emirato", in Mil anos de Fortificaçôes na Península Ibérica e no Magreb (500-1500), Ediçoes Colibri, Lisboa, pp. 59-75.

Aguilella, G.; Falomir, F.; Pérez, G.; Laguna, M.; García, D.A.; Arquer, N. (2016). "Tossal de la Vila (Serra d'en Galceran, Castellón). Un asentamiento en la transición del Bronce Final al Hierro Antiguo", Quaderns de Prehistòria i Arqueologia de Castelló, 34, pp. 5-41.

Bazzana, A. (1992). Maisons d'al-Andalus, Casa de Velázquez, Madrid.

Castellanos, S.; Martín-Viso, I. (2005). "The local articulation of central power in the north of the Iberian Peninsula, 500-1000”, Early Medieval Europe, 13 (1), pp. 1-42.

Catalán, R.; Fuentes, P.; Sastre, J.C. (2014). Fortificaciones en la tardoantigüedad: élites y articulación del territorio (siglos V-VIII d.C.), La Ergástula, Madrid.

Gibert, J. (2018). La fi del món antic i els inicis de l'edat mitjana a la Catalunya Central. Economia, societat $i$ territori entre els segles Vi VIII, ICAC, Tarragona.

Gutiérrez, S. (2012). "Gramática de la casa. Perspectivas de análisis arqueológicos de los espacios domésticos medievales en la península ibérica (siglos VII-XIII)”, Arqueología de la Arquitectura, 9, pp. 139-164.

Lorrio, A.J.; Sánchez de Prado, Mª.D. (2008). "El Molón (Camporrobles, Valencia). Un poblado de primera época islámica”, Lvcentvm, 27, pp. 141-164.

Negre, J. (2013). "Evolució de la xarxa viària del territori de Tortosa entre l'Antiguitat i l'Edat Mitjana”, Quaderns de Prehistòria i Arqueologia de Castelló, 31, pp. 209-226.

Negre, J.; Falomir, F.; Pérez-Polo, M.; Blasco, M.; Aguilella, G.; Medina, P. (en prensa). "Una lectura contextual del recinto emiral del Tossal de la Vila", in Gutiérrez, S.; Amorós, V.; Doménech, C., ed., El sitio de las cosas: la Alta Edad Media en contexto, Universitat d'Alacant, Alacant.

Negre, J.; Suñé, J. (2019). “Territorio, fiscalidad y actividad militar en la formación de un espacio fronterizo. La consolidación de Tortosa como límite extremo del al-Andalus Omeya”, Anuario de Estudios Medievales, 49 (2), pp. 705-740.

Schneider, L. (2001). “Oppida et castra tardo-antiques. À propos des établissements de hauteur de la Gaule Méditerranéenne”, in Ouzoulias, P.; Pellecuer, C.; Rayunaud, C.; Ossel, P. van.; Garmy, P., ed., Les campagnes de la Gaule à la fin de l'Antiquité, APDCA, Antibes, pp. 433-448. 